\title{
A New Set of Stability Criteria Extending Lyapunov's Direct Method
}

\author{
William Li, Delbarton School
}

January 7, 2020

\begin{abstract}
A dynamical system is a mathematical model described by a high dimensional ordinary differential equation for a wide variety of real world phenomena, which can be as simple as a clock pendulum or as complex as a chaotic Lorenz system. Stability is an important topic in the studies of the dynamical system. A major challenge is that the analytical solution of a time-varying nonlinear dynamical system is in general not known. Lyapunov's direct method is a classical approach used for many decades to study stability without explicitly solving the dynamical system, and has been successfully employed in numerous applications ranging from aerospace guidance systems, chaos theory, to traffic assignment. Roughly speaking, an equilibrium is stable if an energy function monotonically decreases along the trajectory of the dynamical system. This paper extends Lyapunov's direct method by allowing the energy function to follow a rich set of dynamics. More precisely, the paper proves two theorems, one on globally uniformly asymptotic stability and the other on stability in the sense of Lyapunov, where stability is guaranteed provided that the evolution of the energy function satisfies an inequality of a non-negative Hurwitz polynomial differential operator, which uses not only the first-order but also high-order time derivatives of the energy function. The classical Lyapunov theorems are special cases of the extended theorems. the paper provides an example in which the new theorem successfully determines stability while the classical Lyapunov's direct method fails.
\end{abstract}

\section{Introduction}

Consider a continuous time dynamical system

$$
\dot{x}=f(x, t)
$$


where $x \in \mathbb{R}^{n}$ is a point in an $n$-th dimensional space and $t$ is a one dimensional variable representing time. $x(t)$ is the state of the dynamical system at time $t$ and represents the trajectory of the point as time passes. The initial state is given as $x\left(t_{0}\right)=x_{0}$. If $f(x, t)$ is only a function of $x$, the system is called time-invariant; otherwise, it is time-varying. Furthermore, if $f(x, t)=A x$ for some square matrix $A$, it is linear time-invariant. The dynamical system is a mathematical model widely used in many disciplines including engineering, physics, economics and biology.

A point $x^{*}$ is an equilibrium point of $(1)$ if $f\left(x^{*}, t\right)=0$ for any $t \geq 0$. If the initial state $x_{0}=x^{*}$, then the state will remain at $x^{*}$ forever. The stability of the equilibrium point is to characterize whether the state will return to $x^{*}$ after certain perturbation away from it or diverge. Stability is an important topic in the studies of the dynamical system. The stability criteria for a linear time-invariant system have been well developed. However, it is difficult to examine the stability of a nonlinear or time-varying system, because the analytical solution of such a system is in general not known.

Lyapunov's direct method, which was founded in A. M. Lyapunov's thesis The General Problem of Stability of Motion at Moscow University in 1892 (Wikipedia, 2018), has been a widely used approach to study the stability of the dynamical system (Parks, 1992). Rather than solving (1) analytically, the method employs a scalar positive definite function $V(x, t)$ intuitively representing the energy of the state, where $V\left(x^{*}, t\right)=0$ and $V(x, t)>0$ for any $x \neq x^{*} \cdot \dot{V}(x, t)$ is the time derivative of $V(x, t)$ along the trajectory. If $\dot{V}(x, t)<0$ for any $x$ except at $x^{*}$, then the energy decreases monotonically over time and the trajectory converges to $x^{*}$. Lyapunov's direct method has been employed in numerous applications ranging from aerospace guidance systems, chaos theory, to traffic assignment (Wikipedia, 2018).

It should be pointed out that Lyapunov's direct method is sufficient but not necessary. Intuitively, for example, the dynamical system is still stable even if the energy does not monotonically decrease as long as it eventually converges to 0 . This paper will develop this idea rigorously and propose a new set of stability criteria, which are more relaxed than the conventional Lyapunov stability criteria. Specifically, the energy function is still employed but does not have to be monotonically decreasing. Instead, the evolution of the energy is to satisfy an inequality of a Hurwitz polynomial differential operator defined in the paper, which uses not only the first-order but also high-order time derivatives of $V(x, t)$.

The remaining of this paper is organized as follows. Section 2 introduces two commonly used definitions of stability, namely stability in the sense of Lyapunov and asymptotic stability, and reviews the Lyapunov's direct method. Section 3 introduces the notion of a Hurwitz polynomial and proposes a definition of nonnegative Hurwitz polynomial differential operator. Section 4 presents the main 
results of this paper, i.e., the new set of stability criteria extending Lyapunov's direct method. Section 5 shows an example in which the new criteria can determine the stability while the conventional Lyapunov stability criteria cannot. The paper is concluded in Section 6 .

\section{Definitions of Stability and Lyapunov's Direct Method}

For the sake of simplifying the description, shift the origin of the system by $-x^{*}$ so that the equilibrium point is at $x^{*}=0$. The following two definitions of stability are commonly used (Teschl, 2012).

\section{Definition 2.1 Stability in the sense of Lyapunov}

The equilibrium point $x^{*}=0$ is stable in the sense of Lyapunov at $t=t_{0}$ if for any $\epsilon>0$ there exists a $\delta\left(t_{0}, \epsilon\right)>0$ such that if $\left\|x_{0}\right\|<\delta\left(t_{0}, \epsilon\right)$ then $\|x(t)\|<\epsilon, \forall t \geq$ $t_{0}$. Furthermore, $x^{*}$ is uniformly stable if $\delta$ is not a function of $t_{0}$.

\section{Definition 2.2 Asymptotic stability}

The equilibrium point $x^{*}=0$ is asymptotically stable at $t=t_{0}$ if it is stable in the sense of Lyapunov and there exists a $\Delta\left(t_{0}\right)>0$ such that if $\left\|x_{0}\right\|<\Delta\left(t_{0}\right)$ then $\lim _{t \rightarrow \infty}\|x(t)\|=0$. Furthermore, $x^{*}$ is uniformly asymptotically stable if it is uniformly stable and $\Delta$ is not a function of $t_{0} . x^{*}$ is globally asymptotically stable if $\lim _{t \rightarrow \infty}\|x(t)\|=0$ for $x_{0}$ anywhere in $\mathbb{R}^{n}$.

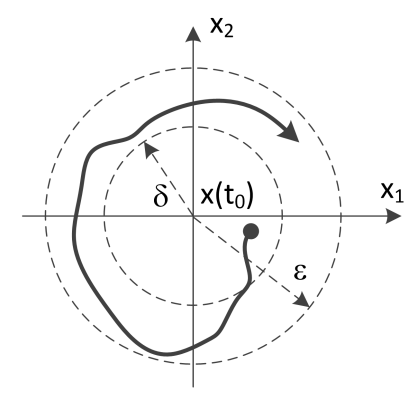

(a) Stability in the sense of Lyapunov

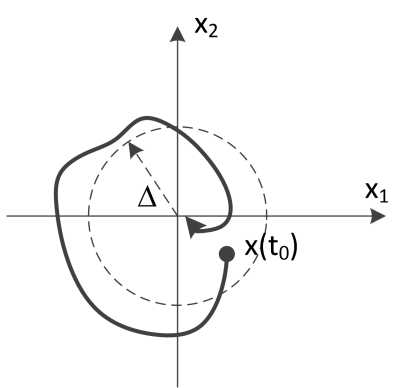

(b) Asymptotic stability

Figure 1: Illustration of two types of local stability. For global asymptotic stability, $\Delta=\infty$. 
Figure 1 illustrates the concepts of the two types of stability. Clearly asymptotic stability is stronger than Lyapunov stability.

Lyapunov's direct method can be used to determine the asymptotic stability of the dynamical system without analytically solving the differential equation (1), as stated in the following theorem (Narendra \& Annaswamy, 1989).

\section{Theorem 2.3 Lyapunov theorem for global uniform asymptotic stability}

The dynamical system (1) is globally uniformly asymptotically stable if a scale function $V(x, t)$ with continuous partial derivative with respect to $x, t$ exists and if the following conditions are satisfied:

1. There exists continuous non-descending functions $\alpha(\|x\|)$ and $\beta(\|x\|)$, such that $\forall t \geq t_{0},\|x\|>0$,

$$
\begin{aligned}
& 0<\alpha(\|x\|) \leq V(x, t) \leq \beta(\|x\|), \\
& \alpha(0)=\beta(0)=0, V(0, t)=0, \\
& \alpha(\|x\|) \rightarrow \infty \text { with }\|x\| \rightarrow \infty .
\end{aligned}
$$

2. There exists a continuous non-descending function $W(\|x\|)$ such that $\forall t \geq$ $t_{0},\|x\| \geq 0$,

$$
\begin{aligned}
& \left.\dot{V}(x, t)\right|_{\dot{x}=f(x, t)} \leq-W(\|x\|), \\
& W(\|x\|)>0, \forall\|x\|>0, \text { and } W(0)=0 .
\end{aligned}
$$

Here the time derivative is given by

$$
\left.\dot{V}(x, t)\right|_{\dot{x}=f(x, t)}=\frac{\partial V(x, t)}{\partial t}+\frac{\partial V(x, t)}{\partial x} f(x, t) .
$$

The above two sets of conditions are illustrated in Figure 2.

Similarly the following theorem (Murray, Li, \& Sastry, 1994) states Lyapunov's direct method to determine the stability in the sense of Lyapunov.

\section{Theorem 2.4 Lyapunov theorem for uniform stability in the sense of Lya- punov}

The dynamical system (1) is uniformly stable in the sense of Lyapunov if a scale function $V(x, t)$ with continuous partial derivative with respect to $x, t$ exists and if the following conditions are satisfied: 


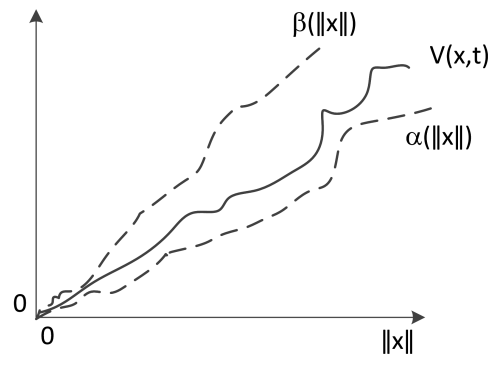

(a) Energy function

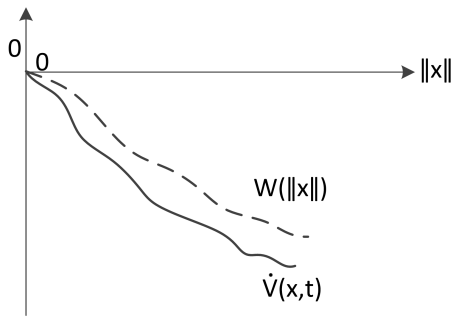

(b) Time derivative of energy function

Figure 2: Illustration of conditions on $V(x, t)$ and $\dot{V}(x, t)$ in the stability theorems of Lyapunov. The energy function $V(x, t)$ is bounded by $\alpha(\|x\|)$ below and $\beta(\|x\|)$ above. The time derivative $\left.\dot{V}(x, t)\right|_{\dot{x}=f(x, t)}$ is bounded by $-W(\|x\|)$ above.

1. There exists continuous non-descending functions $\alpha(\|x\|)$ and $\beta(\|x\|)$ and some constant $\Omega$, such that $\forall t \geq t_{0}, 0<\|x\|<\Omega$,

$$
\begin{aligned}
& 0<\alpha(\|x\|) \leq V(x, t) \leq \beta(\|x\|), \\
& \alpha(0)=\beta(0)=0, V(0, t)=0, \\
& \alpha(\|x\|) \rightarrow \infty \text { with }\|x\| \rightarrow \infty .
\end{aligned}
$$

2. There exists a continuous non-descending function $W(\|x\|)$ such that $\forall t \geq$ $t_{0},\|x\|<\Omega$,

$$
\begin{aligned}
& \left.\dot{V}(x, t)\right|_{\dot{x}=f(x, t)} \leq-W(\|x\|), \\
& W(\|x\|) \geq 0, \text { and } W(0)=0 .
\end{aligned}
$$

The main difference between the two theorems lies in (6) and (12). To ensure asymptotic stability, $W(\|x\|)$ is strictly positive except at $\|x\|=0$. For the stability in the sense of Lyapunov, $W(\|x\|)$ only needs to be non-negative. In addition, conditions (2), (5) and (6) are satisfied globally for $x \in \mathbb{R}^{n}$, while (8), (11) and (12) are satisfied locally near the equilibrium point $\|x\|<\Omega$.

Because $x(t)$ is a function of $t, V(x, t)$ along the trajectory is a function of only $t$. For example, for asymptotic stability, if the conditions (2) to (6) are met, $V(x(t), t)$ monotonically decreases to 0 because the time derivative is strictly negative unless $\|x(t)\|=0$. The time evolution of $V(x(t), t)$ in the two stability theorems of Lyapunov is illustrated in Figure 3(a). 


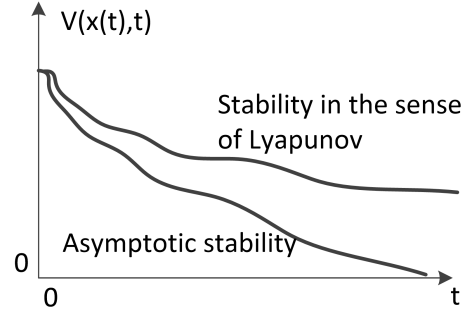

(a)

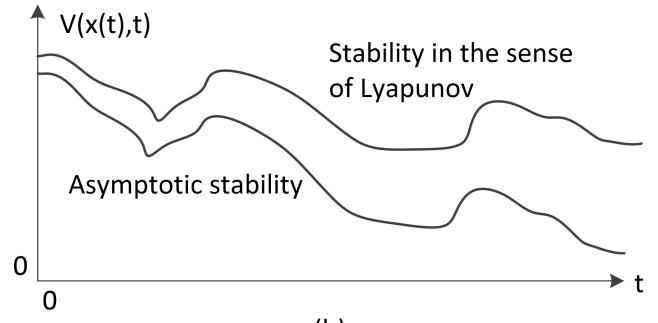

(b)

Figure 3: Illustration of time evolution of $V(x(t), t)$ in the two stability theorems of Lyapunov.

Note that monotonic decrease in $V(x(t), t)$ is sufficient but not necessary to ensure the convergence of $V(x(t), t)$ to 0 or some constant. Figure 3(b) shows two examples. In one example, $V(x(t), t)$ goes up and down and converges to 0 and in the other example $V(x(t), t)$ does not even converge to any constant although it does not diverge either. The main contribution of the paper is to characterize such dynamics of the energy function $V(x(t), t)$. To this end, the next section defines a non-negative Hurwitz polynomial differential operator.

\section{Non-negative Hurwitz Polynomial Differential Oper- ator}

Denote differential operator $D=\frac{d}{d t}$. An $m$-th degree polynomial differential operator is given by

$$
P(D)=\left(\frac{d}{d t}\right)^{m}+a_{1}\left(\frac{d}{d t}\right)^{m-1}+\cdots+a_{m},
$$

where the constant coefficients $a_{1}, \ldots, a_{m}$ are real. In the special case of $m=0$, $a_{0}=1$. The corresponding polynomial is

$$
P(s)=s^{m}+a_{1} s^{m-1}+\cdots+a_{m} .
$$

Denote $\lambda_{1}, \ldots, \lambda_{l}$ the complex roots of polynomial $P(s)$ and $n_{1}, \ldots, n_{l}$ the corresponding multiplicities where $\sum_{j=1}^{l} n_{j}=m . \quad l$ is the number of distinct complex roots.

A time-invariant linear ordinary differential equation

$$
\frac{d^{m} y(t)}{d t^{m}}+a_{1} \frac{d^{m-1} y(t)}{d t^{m-1}}+\cdots+a_{m} y(t)=z(t)
$$




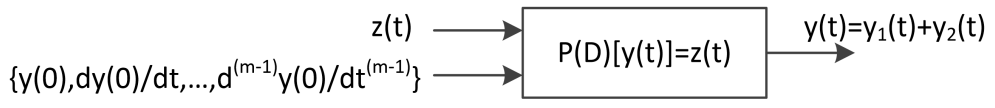

Figure 4: Illustration of input and output of a linear system corresponding to linear differential equation $P(D)[y(t)]=z(t)$.

can be written in the form of a polynomial differential operator

$$
P(D)[y(t)]=z(t) .
$$

This linear differential equation is said to be defined by $P(D) . y(t)$ and $z(t)$ are called the output and input of the corresponding linear system, respectively, as shown in Figure 4.

The solution of (15) or (16) with input $z(t)$ and initial conditions $y(0), \frac{d y(0)}{d t}$, $\ldots, \frac{d^{m-1} y(0)}{d t^{m-1}}$ is given by (Lathi, 2005)

$$
y(t)=y_{1}(t)+y_{2}(t)
$$

where $y_{1}(t)$ is the zero-state response and $y_{2}(t)$ is the zero-input response given by

$$
y_{2}(t)=\sum_{j=1}^{l}\left(\sum_{k=1}^{n_{j}} c_{k, j} t^{k-1}\right) e^{\lambda_{j} t} .
$$

For unit step input function

$$
z(t)= \begin{cases}1, & \text { if } t \geq 0 \\ 0, & \text { otherwise }\end{cases}
$$

$y_{1}(t)$ is called the zero-state unit step response and given by

$$
y_{1}(t)=\frac{1}{a_{m}}\left(1-\sum_{j=1}^{l}\left(\sum_{k=1}^{n_{j}} d_{k, j} t^{k-1}\right) e^{\lambda_{j} t}\right),
$$

Constant coefficients $d_{k, j}$ are completely determined by $P(D) . c_{k, j}$ is a function of both $P(D)$ and the initial conditions $y(0), \frac{d y(0)}{d t}, \ldots, \frac{d^{m-1} y(0)}{d t^{m-1}}$.

The notion of zero-state unit step response is meaningful only when $a_{m} \neq 0$, which holds for any Hurwitz polynomial differential operator $P(D)$ defined next. 


\section{Definition 3.1 Hurwitz polynomial and Hurwitz polynomial differential operator (Kuo, 1966)}

Polynomial $P(s)$ is said to be Hurwitz if the roots of $P(s), \lambda_{1}, \ldots, \lambda_{l}$, all have negative real parts. Polynomial differential operator $P(D)$ is Hurwitz if the corresponding polynomial $P(s)$ is Hurwitz.

If $P(D)$ is Hurwitz, then $a_{m} \neq 0$, because otherwise $P(s)$ has a root at $\lambda=0$.

It follows from (20) and (18) that if (16) is Hurwitz, then

$$
\begin{aligned}
& \lim _{t \rightarrow \infty} y_{1}(t)=\frac{1}{a_{m}}, \\
& \lim _{t \rightarrow \infty} y_{2}(t)=0 .
\end{aligned}
$$

Lemma 3.2 $\int_{t_{0}}^{t} y_{1}(\tau) d \tau$ goes to infinity as $t \rightarrow \infty$ for any fixed $t_{0}$.

Proof This follows immediately from (21).

I next define a new type of Hurwitz polynomial differential operator, which has not been studied in the literature so far, but will be used in Section 4 .

\section{Definition 3.3 Non-negative Hurwitz polynomial differential operator}

Hurwitz polynomial differential operator $P(D)$ is non-negative if the zero-state unit step response defined in (20), $y_{1}(t) \geq 0, \forall t \geq 0$.

Lemma 3.4 Any Hurwitz polynomial differential operator $P(D)$ for $m=0,1,2,3$ is non-negative.

Proof Solve the $m$ coefficients $d_{k, j}$ of the zero-state unit step response (20) by setting the initial conditions to zero

$$
y(0)=\frac{d y(0)}{d t}=\ldots=\frac{d^{m-1} y(0)}{d t^{m-1}}=0 .
$$

For $m=0,(15)$ is reduced to $y(t)=z(t)$. Thus $y_{1}(t)=1 . P(D)$ is non-negative.

For $m=1, \frac{d y(t)}{d t}+a_{1} y(t)=z(t)$. For the differential operator to be Hurwitz, $a_{1}>0$. The zero-state unit step response is

$$
y_{1}(t)=\frac{1}{a_{1}}\left(1-e^{-a_{1} t}\right) \geq 0, \forall t \geq 0 .
$$


For $m=2, \frac{d^{2} y(t)}{d t^{2}}+a_{1} \frac{d y(t)}{d t}+a_{2} y(t)=z(t)$. For the differential operator to be Hurwitz, $a_{2}>0$. There are three cases of the roots of $P(s)$.

- First, distinct real roots $\lambda_{1}, \lambda_{2}<0$.

$$
y_{1}(t)=\frac{1}{a_{2}}\left(1-\frac{\lambda_{2}}{\lambda_{2}-\lambda_{1}} e^{\lambda_{1} t}-\frac{\lambda_{1}}{\lambda_{1}-\lambda_{2}} e^{\lambda_{2} t}\right) .
$$

- Second, distinct complex roots $\lambda_{1,2}=\sigma \pm \omega i$ with $\sigma<0$.

$$
y_{1}(t)=\frac{1}{a_{2}}\left(1-\left(\cos (\omega t)-\frac{\sigma}{\omega} \sin (\omega t)\right) e^{\sigma t}\right) \text {. }
$$

- Third, identical real root $\lambda_{1}<0$.

$$
y_{1}(t)=\frac{1}{a_{2}}\left(1-\left(1-\lambda_{1} t\right) e^{\lambda_{1} t}\right) .
$$

The minimum value of $y_{1}(t)$ can be obtained by letting $\dot{y}_{1}(t)=0$. It can be verified by algebra that the minimum value is non-negative in all of the three cases. Therefore, $y_{1}(t) \geq 0, \forall t \geq 0$,

For $m=3, \frac{d^{3} y(t)}{d t^{3}}+a_{1} \frac{d^{2} y(t)}{d t^{2}}+a_{2} \frac{d y(t)}{d t}+a_{3} y(t)=z(t)$. For the differential operator to be Hurwitz, $a_{3}>0$. There are four cases of the roots of $P(s)$.

- First, distinct real roots $\lambda_{1}, \lambda_{2}, \lambda_{3}<0$.

$$
\begin{aligned}
& y_{1}(t)=\frac{1}{a_{3}}\left(1-d_{1} e^{\lambda_{1} t}-d_{2} e^{\lambda_{2} t}-d_{3} e^{\lambda_{3} t}\right), \\
& d_{1}=\frac{\lambda_{2} \lambda_{3}}{\left(\lambda_{2}-\lambda_{1}\right)\left(\lambda_{3}-\lambda_{1}\right)}, d_{2}=\frac{\lambda_{3} \lambda_{1}}{\left(\lambda_{3}-\lambda_{2}\right)\left(\lambda_{1}-\lambda_{2}\right)}, d_{3}=\frac{\lambda_{1} \lambda_{2}}{\left(\lambda_{1}-\lambda_{3}\right)\left(\lambda_{2}-\lambda_{3}\right)} .
\end{aligned}
$$

- Second, a pair of complex roots $\lambda_{1,2}=\sigma \pm \omega i$ with $\sigma<0$ and one real root $\lambda_{3}<0$.

$$
\begin{aligned}
& y_{1}(t)=\frac{1}{a_{3}}\left(1-d_{3} e^{\lambda_{3} t}-b_{\sigma}\left(b_{c} \cos (\omega t)-b_{s} \sin (\omega t)\right) e^{\sigma t}\right), \\
& d_{3}=\frac{\sigma^{2}+\omega^{2}}{\left(\sigma-\lambda_{3}\right)^{2}+\omega^{2}}, b_{\sigma}=\frac{\lambda_{3}}{\omega\left(\left(\lambda_{3}-\sigma\right)^{2}+\omega^{2}\right)}, \\
& b_{c}=\left(\omega \lambda_{3}-2 \sigma \omega\right), b_{s}=\left(\lambda_{3} \sigma-\sigma^{2}+\omega^{2}\right) .
\end{aligned}
$$




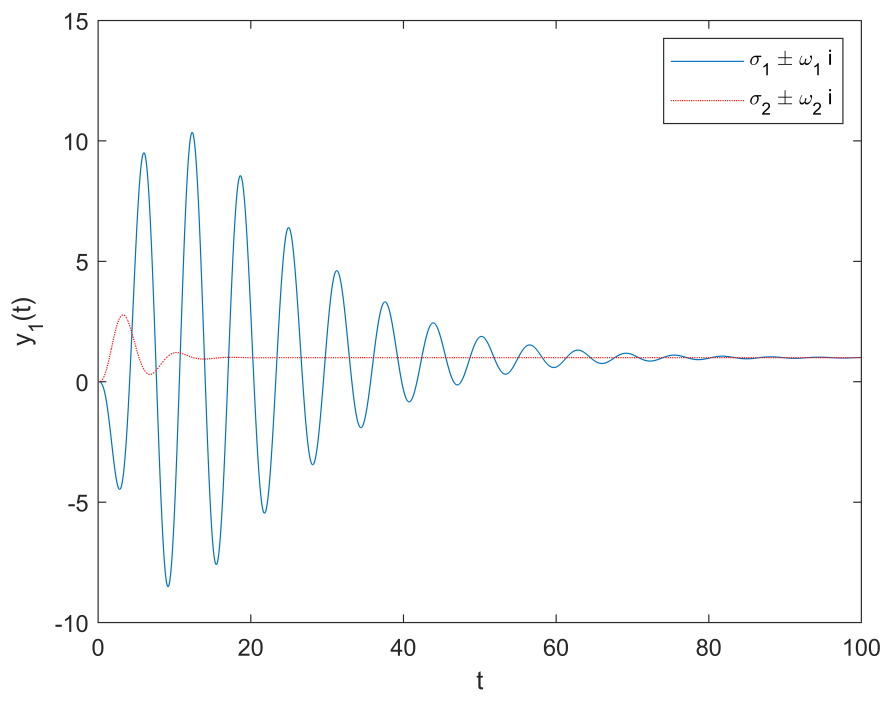

Figure 5: Zero-state unit step response $y_{1}(t)$ defined in (20) of two 4-th order Hurwitz polynomial differential operators.

- Third, a pair of identical real roots $\lambda_{1}<0$ and one distinct real root $\lambda_{2}<0$.

$$
\begin{aligned}
& y_{1}(t)=\frac{1}{a_{3}}\left(1-\left(d_{1,1}+d_{2,1} t\right) e^{\lambda_{1} t}-d_{2} e^{\lambda_{2} t}\right) \\
& d_{1,1}=\frac{\lambda_{2}^{2}-2 \lambda_{1} \lambda_{2}}{\lambda_{1}^{2}-2 \lambda_{1} \lambda_{2}+\lambda_{2}^{2}}, d_{2,1}=\frac{\lambda_{1} \lambda_{2}}{\lambda_{1}-\lambda_{2}}, d_{2}=\frac{\lambda_{1}^{2}}{\lambda_{1}^{2}-2 \lambda_{1} \lambda_{2}+\lambda_{2}^{2}} .
\end{aligned}
$$

- Fourth, three identical real roots $\lambda_{1}<0$.

$$
y_{1}(t)=\frac{1}{a_{3}}\left(1-\left(1-\lambda_{1} t+\frac{\lambda_{1}^{2}}{2} t^{2}\right) e^{\lambda_{1} t}\right)
$$

Similarly to the case of $m=2, y_{1}(t) \geq 0, \forall t \geq 0$, in all of the four cases.

It should be pointed out that not all Hurwitz polynomial differential operators are non-negative. Figure 5 shows the zero-state unit step response $y_{1}(t)$ of $m=4$ where polynomial $P(s)$ has a pair of complex roots $\sigma_{1} \pm \omega_{1} i=\frac{-0.1 \pm i}{\sqrt{(-0.1)^{2}+1^{2}}}$ each with multiplicity 2 . It is clear that while $y_{1}(t)$ converges, $y_{1}(t)<0$ for some $t$. This is an example where $P(D)$ is not non-negative. As the damping factor increases, the polynomial differential operator can be made non-negative. Figure 5 shows that $y_{1}(t)>0, \forall t$ when the complex roots become $\sigma_{2} \pm \omega_{2} i=\frac{-0.5 \pm i}{\sqrt{(-0.5)^{2}+1^{2}}}$. 


\section{Main Results}

The higher order time derivatives of $V(x, t)$ along the trajectory can be derived from (7). For example, the second order time derivative is given by

$$
\left.\frac{d^{2} V(x, t)}{d t^{2}}\right|_{\dot{x}=f(x, t)}=\frac{\partial^{2} V}{\partial t^{2}}+2 \frac{\partial^{2} V}{\partial x \partial t} f+\frac{\partial^{2} V}{\partial x^{2}} f^{2}+\frac{\partial V}{\partial x} \frac{\partial f}{\partial x} f+\frac{\partial V}{\partial x} \frac{\partial f}{\partial t} .
$$

For $\frac{d^{2} V(x, t)}{d t^{2}}$ to exist, all the second order partial derivatives of $V$ with respect to $x, t$ have to exist and the first order partial derivatives of $f$ with respect to $x, t$ have to exist. In general, for the $m$-th order time derivative $\frac{d^{m} V(x, t)}{d t^{m}}$ to exist, all the $m$-th order partial derivatives of $V$ with respect to $x, t$ have to exist and the $(m-1)$-th order partial derivatives of $f$ with respect to $x, t$ have to exist.

\section{Theorem 4.1 Extended theorem for global uniform asymptotic stability}

The dynamical system (1) is globally asymptotically uniformly stable if a scale function $V(x, t)$ with continuous $(m+1)$-th order time derivative along the trajectory exists such that

1 .

$$
\max _{q=1, \ldots, m}\left|\frac{d^{(q)} V\left(x, t_{0}\right)}{d t^{q}}\right| \leq \gamma(\|x\|),
$$

where $\gamma(\|x\|)$ is a continuous non-descending function,

2. $\dot{V}(x, t)$ along the trajectory given by (7) is bounded above by some positive function $\rho(\|x\|)$ when $t$ is sufficiently large,

$$
\dot{V}(x, t) \leq \rho(\|x\|)
$$

i.e., $\dot{V}(x, t)$ does not diverge to $+\infty$ as $t \rightarrow \infty$ as long as $x(t)$ is finite, which is a much weaker assumption than (5) and (6),

and if the conditions in Theorem 2.3 are satisfied except that (5) is replaced by

$$
\left.P(D)[\dot{V}(x, t)]\right|_{\dot{x}=f(x, t)} \leq-W(\|x\|),
$$

where $P(D)$ is an $m$-th degree non-negative Hurwitz polynomial differential operator. 


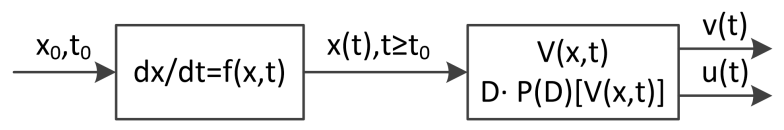

(a)

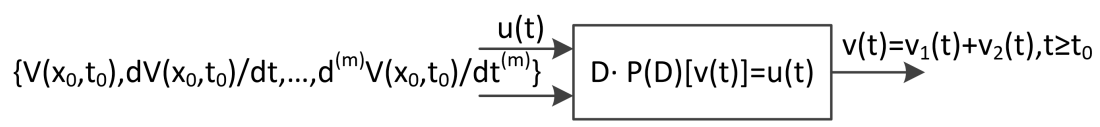

(b)

Figure 6: Two equivalent system representations. (a) is a conventional representation using the time-varying nonlinear dynamical system (1). (b) is an equivalent representation using a time-invariant linear ordinary differential operator $D \cdot P(D)$.

Proof Along the trajectory of $(1),\left.V(x, t)\right|_{\dot{x}=f(x, t)}$ is a function of $t$. Denote

$$
\begin{aligned}
v(t) & =\left.V(x, t)\right|_{\dot{x}=f(x, t)}, \\
u(t) & =\left.P(D)[\dot{V}(x, t)]\right|_{\dot{x}=f(x, t)} \\
& =\left.(D \cdot P(D))[V(x, t)]\right|_{\dot{x}=f(x, t)} .
\end{aligned}
$$

From the assumption of the theorem, $v(t) \geq 0, u(t) \leq 0, \forall t$.

Equivalently, $u(t)$ and $v(t)$ can be considered the input and output, respectively, of a time-invariant linear ordinary differential equation defined by the $(m+1)$-th degree polynomial differential operator $D \cdot P(D)$. Thus, similar to $(17), v(t)$ can be written in two independent parts

$$
v(t)=v_{1}(t)+v_{2}(t)
$$

where $v_{1}(t)$ is the zero-state response of the differential equation with $u(t)$ as the input and

$$
\left\{v_{1}\left(t_{0}\right), \dot{v}_{1}\left(t_{0}\right), \ldots, v_{1}^{(m)}\left(t_{0}\right)\right\}=\{0,0, \ldots, 0\},
$$

and $v_{2}(t)$ is the zero-input response with the initial conditions given by

$$
\left\{v_{2}\left(t_{0}\right), \dot{v}_{2}\left(t_{0}\right), \ldots, v_{2}^{(m)}\left(t_{0}\right)\right\}=\left\{V\left(x_{0}, t_{0}\right), \dot{V}\left(x_{0}, t_{0}\right), \ldots, V^{(m)}\left(x_{0}, t_{0}\right)\right\},
$$

where the time derivatives $\dot{V}, \ldots, V^{(m)}$ are taken along the trajectory. The above two equivalent representations are illustrated in Figure 6. 


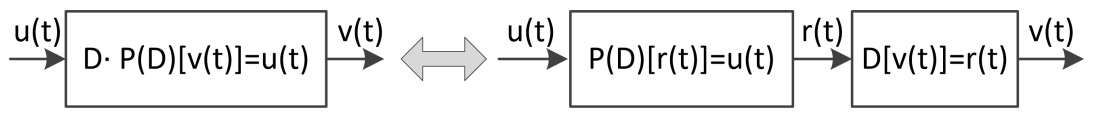

Figure 7: Linear system of $D \cdot P(D)$ is equivalent to two cascaded linear systems $P(D)$ and $D$.

Furthermore, the linear system of $D \cdot P(D)[v(t)]=u(t)$ is equivalent to two cascaded linear systems $P(D)[r(t)]=u(t)$ and $D[v(t)]=r(t)$ as shown in Figure 7 . Therefore, $v_{2}(t)$ is given by

$$
\begin{aligned}
v_{2}(t) & =\int_{t_{0}}^{t} r_{2}\left(\tau-t_{0}\right) d \tau+V\left(x_{0}, t_{0}\right) \\
& =\int_{t_{0}}^{t} \sum_{j=1}^{l}\left(\sum_{k=1}^{n_{j}} c_{k, j}\left(\tau-t_{0}\right)^{k-1}\right) e^{\lambda_{j}\left(\tau-t_{0}\right)} d \tau+V\left(x_{0}, t_{0}\right),
\end{aligned}
$$

where $r_{2}$ is the zero-input response of the linear system $P(D)[r(t)]=u(t)$ and is given in (18). As noted previously, $\lambda_{j}, n_{j}$ depend on $P(D)$ and coefficients $c_{k, j}$ depend on $P(D)$ and the initial conditions $\left\{\dot{V}\left(x_{0}, t_{0}\right), \ldots, V^{(m)}\left(x_{0}, t_{0}\right)\right\}$. Because $P(D)$ is Hurwitz, the real part of $\lambda_{j}$ is negative for any $j$. Therefore, $v_{2}(t)$ converges to some constant as $t \rightarrow \infty$.

Because $v(t) \geq 0, \forall t$, it follows that

$$
v_{1}(t)=v(t)-v_{2}(t)>-M, \text { for } t>T,
$$

where $\mathrm{T}$ and $\mathrm{M}$ are large positive constants. Denote $h(t)$ the zero-state unit impulse response of the linear differential equation defined by $P(D)$. From the property of impulse response, the zero-state response of this linear differential equation with $u(t)$ as the input is given by

$$
r_{1}(\xi)=\int_{t_{0}}^{\xi} u(\tau) h(\xi-\tau) d \tau .
$$

$v_{1}$, the zero-state response of the linear differential equation defined by $D \cdot P(D)$, is the integral of $r_{1}(\xi)$ over $\xi$

$$
\begin{aligned}
v_{1}(t) & =\int_{t_{0}}^{t} r_{1}(\xi) d \xi \\
& =\int_{t_{0}}^{t} \int_{t_{0}}^{\xi} u(\tau) h(\xi-\tau) d \tau d \xi \\
& =\int_{t_{0}}^{t}\left(\int_{0}^{t-\tau} h(\eta) d \eta\right) u(\tau) d \tau \\
& =\int_{t_{0}}^{t} y_{1}(t-\tau) u(\tau) d \tau
\end{aligned}
$$


where $y_{1}(\cdot)$ is the zero-state unit step response and is given in (20). The last step holds because $\int_{0}^{t-\tau} h(\eta) d \eta$ is the unit step response of the linear differential equation defined by $P(D)$ at the instant of $t-\tau$, i.e.,

$$
\int_{0}^{t-\tau} h(\eta) d \eta=y_{1}(t-\tau)
$$

Because $P(D)$ is non-negative, $y_{1}(t-\tau)$ is non-negative for any $t-\tau$, and because $u(\tau) \leq 0, \forall \tau$,

$$
y_{1}(t-\tau) u(\tau) \leq 0 .
$$

I next show by contradiction that

$$
\lim _{t \rightarrow \infty} v(t)=0
$$

Assume that $v(t)$ does not converge to 0 as $t \rightarrow \infty$. Then there exist a positive constant $\epsilon$ and an infinite time sequence $\left\{t_{i}\right\}, i=1,2, \ldots$, such that $v\left(t_{i}\right)>\epsilon$, where $t_{i+1}>t_{i}$ and $t_{i}$ goes to infinite as $i \rightarrow \infty$. From (2),

$$
\beta\left(\left\|x\left(t_{i}\right)\right\|\right) \geq \epsilon
$$

Because $\beta(\cdot)$ is continuous and non-descending, there must exist a positive constant $\mu(\epsilon)$ as a function of $\epsilon$ such that

$$
\left\|x\left(t_{i}\right)\right\| \geq \mu(\epsilon)
$$

Furthermore, because $W(\cdot)$ is continuous and non-descending,

$$
W\left(\left\|x\left(t_{i}\right)\right\|\right) \geq \omega(\epsilon),
$$

where $\omega(\epsilon)$ is a positive constant as a function of $\epsilon$. Therefore,

$$
u\left(t_{i}\right) \leq-W\left(\left\|x\left(t_{i}\right)\right\|\right) \leq-\omega(\epsilon) .
$$

If a time instant $t$ exist in interval $\left(t_{i-1}, t_{i}\right)$ such that $v(t)=\frac{\epsilon}{2}$, then let $s_{i}$ be the maximum value of $t$ such that $t<t_{i}$ and $v(t)=\frac{\epsilon}{2}$. Otherwise, let $s_{i}=t_{i-1}$. Figure 8 illustrates the two different cases of $t_{i}$ and $s_{i}$. In either case,

$$
v(t) \geq \frac{\epsilon}{2}, \forall t \in\left[s_{i}, t_{i}\right],
$$

By construction, intervals $\left[s_{i}, t_{i}\right]$ do not overlap each other except possibly at the end points.

I next show that an infinite number $s_{i}$ exist such that $v\left(s_{i}\right)=\frac{\epsilon}{2}$. Assume on the contrary that a number $i_{0}$ exists such that for any $i>i_{0}, s_{i}=t_{i-1}$. This means 


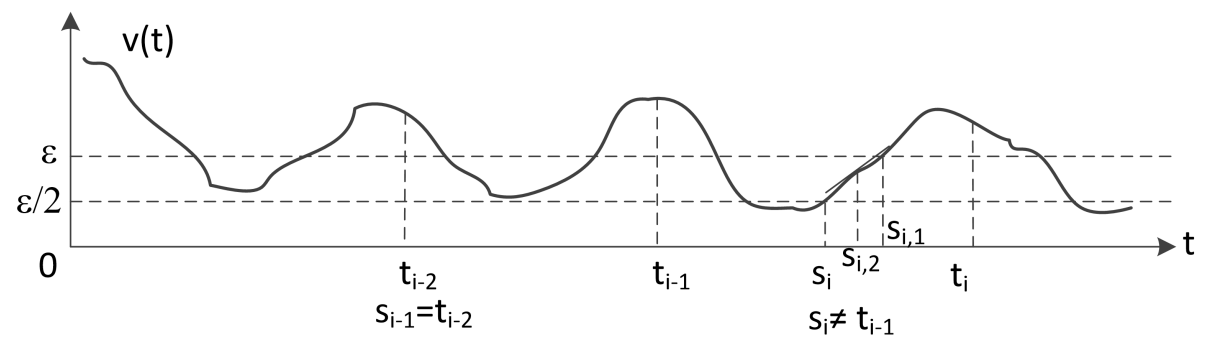

Figure 8: Illustration of $v(t)$ when it does not converge to 0 .

$v(t)>\frac{\epsilon}{2}, \forall t>t_{i_{0}}$. Then $u(t) \leq-\omega\left(\frac{\epsilon}{2}\right)$, where $\omega\left(\frac{\epsilon}{2}\right)$ is a constant derived similarly to $\omega(\epsilon)$ in (44), (45), and (46). From (40) and (42)

$$
\begin{aligned}
v_{1}(t) & \leq \int_{t_{i_{0}}}^{t} y_{1}(t-\tau) u(\tau) d \tau \\
& \leq-\omega\left(\frac{\epsilon}{2}\right) \int_{t_{i_{0}}}^{t} y_{1}(t-\tau) d \tau .
\end{aligned}
$$

From Lemma 3.2, $v_{1}(t)$ goes to negative infinite as $t \rightarrow \infty$, which contradicts (35).

Denote the subsequence $s_{i_{k}}$ for which $v\left(s_{i_{k}}\right)=\frac{\epsilon}{2}$. As shown below by contradiction, $t_{i_{k}}-s_{i_{k}}$ does not converge to 0 as $k \rightarrow \infty$. Because of the continuity of $v(t)$, there exists $s_{i_{k}, 1} \in\left[s_{i_{k}}, t_{i_{k}}\right]$ such that

$$
\begin{aligned}
& v(t) \leq \epsilon, \forall t \in\left[s_{i_{k}}, s_{i_{k}, 1}\right] \\
& v\left(s_{i_{k}, 1}\right)=\epsilon .
\end{aligned}
$$

As illustrated in Figure 8, $s_{i_{k}, 1}$ is the minimum value of $t$ such that $t>s_{i_{k}}$ and $v(t)=\epsilon$. From the mean-value theorem, there exists $s_{i_{k}, 2} \in\left[s_{i_{k}}, s_{i_{k}, 1}\right]$ such that

$$
\dot{v}\left(s_{i_{k}, 2}\right)=\frac{v\left(s_{i_{k}, 1}\right)-v\left(s_{i_{k}}\right)}{s_{i_{k}, 1}-s_{i_{k}}} \geq \frac{\frac{\epsilon}{2}}{t_{i_{k}}-s_{i, k}} .
$$

Assume that $\lim _{i \rightarrow \infty} t_{i_{k}}-s_{i_{k}}=0$. Then $\dot{v}\left(s_{i_{k}, 2}\right)$ goes to $+\infty$. On the other hand, from $(51)$,

$$
v\left(s_{i_{k}, 2}\right)<\epsilon
$$

From (2),

$$
\left\|x\left(s_{i_{k}, 2}\right)\right\|<K_{1}(\epsilon)
$$

where $K_{1}(\epsilon)$ is a positive constant as a function of $\epsilon$. Thus,

$$
\dot{v}\left(s_{i_{k}, 2}\right)<K_{2}(\epsilon)
$$

for some positive constant $K_{2}(\epsilon)$, because $\dot{V}(x, t)$ along the trajectory is bounded by $\rho(\|x\|)$ when $t$ is sufficiently large from (25) in the theorem. Contradiction! 
Therefore, $t_{i_{k}}-s_{i_{k}}$ does not converge to 0 as $k \rightarrow \infty$. In other words, there exist a positive constant $\zeta$ and a subsequence of $\left\{s_{i_{k}}, t_{i_{k}}\right\}$, referred to as $\left\{s_{i_{k_{j}}}, t_{i_{k_{j}}}\right\}$, $j=1,2, \ldots$, such that

$$
t_{i_{j}}-s_{i_{k_{j}}} \geq \zeta
$$

Denote $\Phi$ the union of all the intervals $\left[s_{i_{k_{j}}}, t_{i_{k_{j}}}\right]$. Similar to (50),

$$
\begin{aligned}
v_{1}(t) & \leq \int_{\Phi} y_{1}(t-\tau) u(\tau) d \tau \\
& \leq-\omega\left(\frac{\epsilon}{2}\right) \int_{\Phi} y_{1}(t-\tau) d \tau .
\end{aligned}
$$

From $(21), y_{1}(t) \geq\left(\frac{1}{a_{m}}-\epsilon_{1}\right)$ for a small positive constant $\epsilon_{1}$ when $t$ is sufficiently large. Therefore, as $t \rightarrow \infty$,

$$
v_{1}(t) \leq-\omega\left(\frac{\epsilon}{2}\right) \sum_{j=1}^{\infty}\left(t_{i_{k_{j}}}-s_{i_{k_{j}}}\right)\left(\frac{1}{a_{m}}-\epsilon_{1}\right) .
$$

Because of (57), the right side and thus $v_{1}(t)$ go to negative infinite, which contradicts (35). Hence, by contradiction, I have proved (43). From (2), I conclude that

$$
\lim _{t \rightarrow \infty}\|x(t)\|=0 .
$$

Note that (61) holds for any $x_{0}, t_{0}$.

Next I show that $x^{*}$ is uniformly stable in the sense of Lyapunov. For any given $\epsilon>0$, to show that $\|x(t)\|<\epsilon, \forall t \geq t_{0}$, from (2) it suffices to require that

$$
v(t)=V(x, t)<\psi(\epsilon), \forall t
$$

where $\psi(\epsilon)$ is a positive constant as a function of $\epsilon$, because $\alpha(\|x\|) \leq V(x, t)<\psi(\epsilon)$ and $\alpha(\|x\|)$ is continuous non-descending and $\alpha(\|x\|)=0$ only when $\|x\|=0$. From (40) and (42),

$$
v_{1}(t) \leq 0, \forall t .
$$

Therefore, it suffices to require that

$$
v_{2}(t)=v(t)-v_{1}(t)<\psi(\epsilon), \forall t
$$

From $(34), v_{2}(t)$ is a linear combination of coefficients $c_{k, j}$ and $V\left(x_{0}, t_{0}\right)$,

$$
v_{2}(t)=\sum_{j=1}^{l} \sum_{k=1}^{n_{j}} c_{k, j}\left(\int_{t_{0}}^{t}\left(\tau-t_{0}\right)^{k-1} e^{\lambda_{j}\left(\tau-t_{0}\right)} d \tau\right)+V\left(x_{0}, t_{0}\right) .
$$


Because $\left(\int_{t_{0}}^{t}\left(\tau-t_{0}\right)^{k-1} e^{\lambda_{j}\left(\tau-t_{0}\right)} d \tau\right)$ converges to some constant as $t \rightarrow \infty$ for any $k, j, \max _{t} \sum_{k, j}\left|\int_{t_{0}}^{t}\left(\tau-t_{0}\right)^{k-1} e^{\lambda_{j}\left(\tau-t_{0}\right)} d \tau\right|$ is bounded and only depends on $P(D)$. Therefore, it suffices to require

$$
\max \left(\max _{k, j}\left|c_{k, j}\right|, V\left(x_{0}, t_{0}\right)\right)<\frac{\psi(\epsilon)}{\max _{t} \sum_{k, j}\left|\int_{t_{0}}^{t}\left(\tau-t_{0}\right)^{k-1} e^{\lambda_{j}\left(\tau-t_{0}\right)} d \tau\right|+1}
$$

The coefficients $c_{k, j}$ are obtained by solving a system of linear algebra equations from (32),

$$
v_{2}^{(q)}\left(t_{0}\right)=\left.\sum_{j=1}^{l} \sum_{k=1}^{n_{j}} c_{k, j} \frac{d^{q}\left(\int_{t_{0}}^{t}\left(\tau-t_{0}\right)^{k-1} e^{\lambda_{j}\left(\tau-t_{0}\right)} d \tau\right)}{d t^{q}}\right|_{t=t_{0}}=V^{(q)}\left(x_{0}, t_{0}\right),
$$

for $q=1,2, \ldots, m$. This system of linear equations can be written in the standard form

$$
A w=b
$$

where vector $w$ consists of coefficients $c_{k, j}$, vector $b$ consists of $V^{(q)}\left(x_{0}, t_{0}\right)$, and the elements of $A$ are $\left.\frac{d^{q}\left(\int_{t_{0}}^{t}\left(\tau-t_{0}\right)^{k-1} e^{\lambda_{j}\left(\tau-t_{0}\right)} d \tau\right)}{d t^{q}}\right|_{t=t_{0}} . A$ is a square because $\sum_{j=1}^{l} n_{j}=$ $m$. Furthermore, for any Hurwitz $P(D), A$ is nonsingular (Lathi, 2005). Thus,

$$
\|w\|_{\infty} \leq\left\|A^{-1}\right\|_{\infty}\|b\|_{\infty} .
$$

Therefore, to satisfy (66), which is an upper bound of $\|w\|_{\infty}$, it suffices to require that

$$
\begin{aligned}
& \max _{q=0, \ldots, m}\left|\frac{d^{(q)} V\left(x_{0}, t_{0}\right)}{d t^{q}}\right|=\|b\|_{\infty} \leq \gamma\left(\left\|x_{0}\right\|\right) \\
& <\left(\frac{\Psi(\epsilon)}{\max _{t} \sum_{k, j}\left|\int_{t_{0}}^{t}\left(\tau-t_{0}\right)^{k-1} e^{\lambda_{j}\left(\tau-t_{0}\right)} d \tau\right|+1}\right) /\left\|A^{-1}\right\|_{\infty},
\end{aligned}
$$

Finally, because $\gamma\left(\left\|x_{0}\right\|\right)$ is a continuous non-descending function, it suffices to require that

$$
\left\|x_{0}\right\|<\delta(\epsilon) .
$$

for some positive $\delta(\epsilon)$, which is independent of $t_{0}$. From Definition 2.1, $x^{*}$ is uniformly stable.

Hence, from Definition $2.2, x^{*}$ is globally asymptotically uniformly stable. 
Theorem 2.3 is a special case of Theorem 4.1 when $m=0$. From Lemma 3.4, one can use any $m=0,1,2,3$ Hurwitz polynomial differential operator. However, it should be pointed out that the use of the high order time derivatives in Theorem 4.1 imposes more stringent requirements on the choice of $V(x, t)$ and on the dynamical system itself. Specifically, from $(7), f(x, t)$ is not necessarily continuous in Theorem 2.3. From (23), both $\frac{\partial f}{\partial x}$ and $\frac{\partial f}{\partial t}$ have to exist to apply Theorem 4.1 with $m=1$. Just like the classical Lynapunov theorem, Theorem 4.1 provides a sufficient condition to test global uniform asymptotic stability. One can try out multiple choices of $m, P(D)$ and $V(x, t)$ to see whether one of them works out.

\section{Theorem 4.2 Extended theorem for uniform stability in the sense of Lya- punov}

The dynamical system (1) is uniformly stable in the sense of Lyapunov if a scale function $V(x, t)$ with continuous $(m+1)$-th order time derivative along the trajectory exists such that (24) holds when $\|x\|<\Omega$, and if the conditions in Theorem 2.4 are satisfied except that (11) is replaced by

$$
\left.P(D)[\dot{V}(x, t)]\right|_{\dot{x}=f(x, t)} \leq-W(\|x\|) .
$$

where $P(D)$ is an $m$-th degree non-negative Hurwitz polynomial differential operator.

Proof Because of the difference between (12) and (6), (46) in the proof of Theorem 4.1 does not hold here and $v(t)$ does not necessarily converge to 0 as shown in (43). The difference between Theorems 4.1 and 4.2 parallels that between Theorems 2.3 and 2.4 .

The proof of Theorem 4.1 has already provided the steps from (62) and onward to show uniform stability. The proof is based on $u(t) \leq 0, \forall t$, which does not rely on (6). (12) is sufficient to ensure $u(t) \leq 0, \forall t$. Therefore, the proof is applicable here, with the only difference being that in this theorem (8), (24) and (72) now hold for $\|x\|<\Omega$ instead of for $x \in \mathbb{R}^{n}$. To show uniform stability here, consider any given $\epsilon>0$. Without loss of generality ${ }^{1}$, suppose that $\epsilon \leq \Omega$. It suffices to show that $\|x(t)\|<\epsilon, \forall t \geq t_{0}$ for which (8), (24) and (72) always hold.

From the proof of Theorem 4.1, it follows that if

$$
\left\|x_{0}\right\|<\min (\delta(\epsilon), \epsilon),
$$

where $\delta(\epsilon)$ is given in (71), then (66) and (70) hold. As a result,

$$
\max _{t \geq t_{0}} v_{2}(t)<\Psi(\epsilon)
$$

\footnotetext{
${ }^{1}$ Otherwise, set $\epsilon=\min (\epsilon, \Omega)$, and find the region of $x_{0}$ for which the trajectory $x(t), \forall t \geq t_{0}$, stays in a smaller region than the original $\epsilon$.
} 
As the trajectory starts with $\left\|x_{0}\right\|<\epsilon \leq \Omega, v_{1}(t) \leq 0$ because $u(t) \leq 0$ from the assumption of the theorem and because of (40) and (42). Thus

$$
V(x, t)=v(t)=v_{1}(t)+v_{2}(t)<\psi(\epsilon),
$$

and therefore $\|x(t)\|<\epsilon$ from (8). In other words, once the trajectory starts within the $\epsilon$ local region around the equilibrium point, (8), (24) and (72) hold, therefore keeping the trajectory to stay within the region.

Hence, it is concluded that $\|x(t)\|<\epsilon, \forall t \geq t_{0}$ and $x^{*}$ is uniformly stable in the sense of Lyapunov from Definition 2.1.

Theorem 2.4 is a special case of Theorem 4.2 when $m=0$.

\section{An Example}

Consider the following example of a one dimensional time-varying linear system

$$
\dot{x}=g(t) x,
$$

with $t_{0} \geq 0$. The solution of this system can be analytically given as

$$
x(t)=x_{0} e^{\int_{t_{0}}^{t} g(\tau) d \tau},
$$

from which stability can be determined. However, for the sake of illustration, I use Lyapunov's direct method and compare the Lyapunov and extended theorems for asymptotic stability.

Let

$$
V(x, t)=\frac{1}{2} x^{2}
$$

It follows that

$$
\dot{V}(x, t)=x \dot{x}=g x^{2} .
$$

To apply Theorem 2.3, $g(t)$ has to be negative $\forall t \geq t_{0}$. Now consider the second order time derivative of $V(x, t)$

$$
\ddot{V}(x, t)=\left(\dot{g}+2 g^{2}\right) x^{2},
$$

and let

$$
P(D)[\dot{V}(x, t)]=\ddot{V}(x, t)+a_{1} \dot{V}(x, t),
$$

where $a_{1}>0$ making $P(D)$ non-negative Hurwitz. It follows that

$$
P(D)[\dot{V}(x, t)]=\left(\dot{g}+2 g^{2}+a_{1} g\right) x^{2}:=G(t) x^{2} .
$$




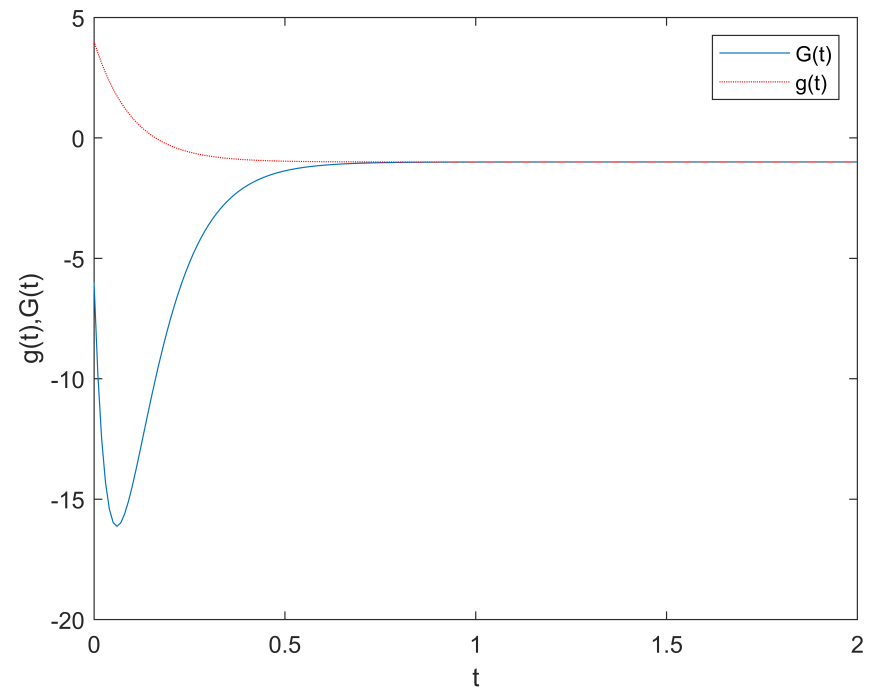

Figure 9: Plots of $g(t), G(t)$ of the dynamical system (77) with (83) with $t_{0}=0$. $\dot{V}(x, t)=g(t) x^{2}$ is not always negative. $P(D)[\dot{V}(x, t)]=G(t) x^{2}<0, \forall t \geq t_{0}$. Therefore the system is globally uniformly asymptotically stable from Theorem 4.1.

To apply Theorem 4.1, $g(t)$ does not have to be negative $\forall t \geq t_{0}$. When $g(t)>0$, if $\dot{g}$ is sufficiently negative, $G(t)$ can be negative. Consider a concrete example of $g(t)$

$$
g(t)=-1+5 e^{-10 t} .
$$

(77) becomes

$$
x(t)=x_{0} e^{t_{0}-t+\frac{1}{2}\left(e^{-10 t_{0}}-e^{-10 t}\right)} .
$$

From Definition $2.2, x^{*}=0$ is globally uniformly asymptotically stable, because

- $\lim _{t \rightarrow \infty} x_{0} e^{t_{0}-t+\frac{1}{2}\left(e^{-10 t_{0}}-e^{-10 t}\right)}=0$.

- $x(t)=x_{0} e^{t_{0}-t+\frac{1}{2}\left(e^{-10 t_{0}}-e^{-10 t}\right)}<x_{0} e^{\frac{1}{2}}, \forall t \geq t_{0}, t_{0} \geq 0$. Thus, for any $\epsilon>0$, let $\delta=\epsilon e^{-\frac{1}{2}}$. If $\left\|x_{0}\right\|<\delta$, then $\|x(t)\|<\epsilon$.

Rather than relying on the analytical solution (84), I now employ Lyapunov's direct method. Let $a_{1}=3$.

$$
G(t)=\dot{g}+2 g^{2}+a_{1} g=-1-55 e^{-10 t}+50 e^{-20 t} .
$$

It is easy to show that $G(t)<0, \forall t \geq t_{0}$. Figure 9 plots $g(t), G(t)$. Because $g(t)$ is not always negative, Theorem 2.3 cannot be applied to confirm global uniform asymptotic stability. Because $G(t)<0, \forall t \geq t_{0}$, Theorem 4.1 can be applied to show that in (77) with (83) $x^{*}=0$ is globally uniformly asymptotically stable. 


\section{Conclusion}

Stability is an important topic in the studies of the dynamical systems. A major challenge is that the analytical solution of a time-varying nonlinear dynamical system is in general not known. Lyapunov's direct method is a classical approach used for many decades to study stability without explicitly solving the dynamical system. Roughly speaking, an equilibrium is stable if an energy function monotonically decreases along the trajectory of the dynamical system. In this paper, I extend Lyapunov's direct method by allowing the energy function to temporarily increase. More precisely, I prove two theorems, one on globally uniformly asymptotic stability and the other on stability in the sense of Lyapunov, where stability is guaranteed provided that the evolution of the energy function satisfies an inequality of a non-negative Hurwitz polynomial differential operator. The classical Lyapunov theorems are special cases of the extended theorems. I provide an example in which the new theorem successfully determines stability while the classical Lyapunov's direct method fails. In the future study I hope to apply the extended stability theorems to more sophisticated dynamical systems in the real world.

\section{References}

Kuo, F. (1966). Network analysis and synthesis (2nd ed.). New York, NY: John Wiley \& Sons.

Lathi, B. P. (2005). Linear systems and signals (2nd ed.). New York, NY: Oxford University Press.

Murray, R. M., Li, Z., \& Sastry, S. S. (1994). A mathematical introduction to robotic manipulation. Boca Raton, FL: CRC Press.

Narendra, K., \& Annaswamy, A. (1989). Stable adaptive systems. Englewood Cliffs, NJ: Prentice-Hall.

Parks, P. C. (1992). A. M. Lyapunov's stability theory - 100 years on. IMA Journal of Mathematical Control ES Information, 9(4), 275 - 303.

Teschl, G. (2012). Ordinary differential equations and dynamical systems (Vol. 140). Providence, RI: American Mathematical Society.

Wikipedia. (2018). Lyapunov stability. Retrieved from https://en.wikipedia .org/wiki/Lyapunov_stability ([Online; accessed 05-January-2018]) 